\title{
Phase separations and nematicity of transition metal impurities
}

\section{Tomasz Diett ${ }^{1,2}$}

\author{
${ }^{1}$ International Research Centre MagTop, Institute of Physics, Polish Academy of Sciences, PL-02668 Warsaw, Poland \\ ${ }^{2}$ WPI-Advanced Institute for Materials Research, Tohoku University, Sendai 980-8577, Japan \\ dietl@MagTop.ifpan.edu.pl
}

\begin{abstract}
Semiconductors [1] and topological materials [2] doped with transition metal elements attract considerable attention due to the fascinating physics and nonospintronic functionalities associated with exchange coupling between band carries and localized spins. However, there is a growing amount of evidence that $d$-shells of magnetic impurities contribute also to bonding, which can affect their spatial distribution and modify key properties, such as magnetic ordering temperature [3]. It has recently been experimentally demonstrated that the resulting phase separation (spinodal decomposition) can be anisotropic and result in the hitherto puzzling rotational symmetry breaking (i.e., nematic characteristics) revealed in a certain class of dilute magnetic semiconductors [4]. This finding put in a new light a possible origin of nematicity in other systems, such as unconventional superconductors and modulation doped semiconductor quantum wells, in which rotational symmetry breaking has so far been assigned to unidirectional spontaneous ordering of spin, orbital or charge degrees of freedom.
\end{abstract}

[1] T. Dietl and H. Ohno, Rev. Mod. Phys. 86, 187-251 (2014).

[2] Y. Tokura, K. Yasuda, and A. Tsukazaki, Nature Rev. Phys. 1, 126-143 (2019).

[3] T. Dietl, K. Sato, T. Fukushima, A. Bonanni, M. Jamet, A. Barski, S. Kuroda, M. Tanaka, Phan Nam Hai, H. Katayama-Yoshida, Rev. Mod. Phys. 87, 1311-1376 (2015).

[4] Ye Yuan, R. Hübner, M. Birowska, Chi Xu, Mao Wang, S. Prucnal, R. Jakieła, K. Potzger, R. Böttger, S. Facsko, J. A. Majewski, M. Helm, M. Sawicki, Shengqiang Zhou, and T. Dietl, Phys. Rev. Materials 2, 114601 (2018).

Keywords: crystallographic phase separation, chemical phase separation, spinodal decomposition, nematicity, dilute magnetic semiconductors

This work has been supported by the Foundation for Polish Science through the IRA Programme financed by te EU within SG OP Programme. 